\title{
A Influência das Condições do Mercado Acionário e da Política Monetária no Comportamento dos Indi- cadores de Risco Tamanho, Índice Book-to-market e Momento, no Mercado Acionário Brasileiro
}

\author{
Adriano Mussa ${ }^{1}$ \\ José Roberto Securato ${ }^{2}$ \\ José Odálio dos Santos ${ }^{3}$ \\ Rubens Famá ${ }^{4}$
}

\section{Resumo}

O presente artigo é um estudo exploratório, em que as variáveis estudadas tNos últimos anos têm se intensificado na literatura nacional e internacional os testes empíricos de modelos APT (ArbitragePricingTheory) para a precificação de ativos, principalmente com o uso de características da empresa para a formação de fatores de risco adicionais ao beta de mercado. Os modelos 3-fatores de Fama \&French e 4-fatores de Carhart são dois exemplos recorrentemente testados desse tipo de modelo, com evidências de relativo sucesso. Diante desse cenário, torna-se importante aprofundar os estudos sobre o comportamento dos fatores de risco que compõem esses modelos. Seguindo o caminho de pesquisas internacionais, o objetivo do presente trabalho é investigar o comportamento dos fatores de risco tamanho, índice book-to-market e momento, no mercado acionário brasileiro, em condições de i) alta e baixa do mercado acionário e ii) expansão e retração monetária. A amostra foi constituída por todas as ações listadas na Bovespa, de junho

\footnotetext{
${ }^{1}$ Mestre em Administração (Finanças) pela Pontifícia Universidade Católica de São Paulo - PUC/SP e Doutorando em Administração (Finanças) pela Faculdade de Economia, Administração e Contabilidade - FEA/USP. Professor da Fundação Instituto de Administração -FIA. End.: Rua Iperoig, 320, apto. 153, Perdizes, São Paulo - SP. CEP: 05016-000 - Brasil. E-mail: adriano.mussa@labfin.com.br.

${ }^{2}$ Livre-Docente em Administração (Finanças) pela Faculdade de Economia, Administração e Contabilidade -FEA/USP. Professor do Departamento de Administração da Universidade de São Paulo - USP. End.: Rua Navarro de Andrade, 152, Pinheiros, São Paulo - SP. CEP: 05418-020 - Brasil.E-mail: securato@usp.br.

${ }^{3}$ Doutor em Administração (Finanças) pela Fundação Getúlio Vargas - FGV/SP. Professor do Departamento de Administração da Pontifícia Universidade Católica de São Paulo - PUC/SP. End.: Rua Monte Alegre, 984, Departamento de Administração, Perdizes, São Paulo - SP. CEP: 05014-901 - Brasil.E-mail: j.odalio@pucsp.br.

${ }^{4}$ Doutor em Administração (Finanças) pela Faculdade de Economia, Administração e Contabilidade - FEA/USP. Professor do Departamento de Administração da Pontifícia Universidade Católica de São Paulo - PUC/SP. End.: Rua Monte Alegre, 984, Departamento de Administração, Perdizes, São Paulo -SP. CEP: 05014-901.E-mail: rfama@usp.br.

Artigo recebido em: 18/04/2010. Artigo aceito em: 12/08/2010. Membro do Corpo Editorial Científico responsável pelo processo editorial: Gilberto de Oliveira Moritz.
}

(c) (i) $\Theta$ Es $\Theta$ Esta obra está sob a Licença Creative Commons Atribuição-Uso. 
de 1995 a junho de 2007. A metodologia de testes empregada foi a mesma utilizada por Fama \&French (1993) na construção das carteiras e fatores de risco. Os resultados indicaram evidências de uma importante influência exercida pelas condições do mercado acionário e pela política monetária sobre a regularidade do comportamento dos fatores de risco no mercado brasileiro.

Palavras-chave: CAPM. APT. Fatores de Risco.

\section{Introdução}

Os modelos de precificação de ativos e os fatores que capturam riscos formam um tema bastante discutido e pesquisado em finanças. Um dos pioneiros no assunto foi Sharpe (1964) que desenvolveu o famoso modelo denominado Capital AssetPricingModel (CAPM), baseado nos trabalhos de Markowitz (1952) e de Tobin (1958). Trata-se de um modelo de fator único, o beta, que seria o fator de explicação da diferença de retorno exigido entre os ativos, em uma relação linear.

Desde então, diversos autores passaram a testar empiricamente a validade do CAPM. Muitos pesquisadores, principalmente nos primeiros estudos, encontraram evidências no sentido de validação do modelo. Entre eles, pode-se citar Black, Jensen e Scholes (1972) e Fama e MacBeth (1973). Porém, o desenvolvimento dos testes trouxe o debate sobre suas deficiências, principalmente devido ao surgimento de novas evidências de que boa parte da variação nos retornos esperados dos ativos não está relacionada ao fator beta de mercado (FAMA; FRENCH, 2004).

Basu (1977), Banz (1981), Stattman (1980), Lakonishok e Shapiro (1986) e Fama eFrench (1992) são exemplos de alguns estudos que identificaram ineficiências do CAPM e terminaram por caracterizar algumas anomalias de mercado, sugerindo a existência de fatores de risco adicionais ao beta.

$\mathrm{Na}$ busca por fatores que pudessem melhorar o poder explicativo do CAPM e capturar anomalias do mercado, Fama eFrench (1993) formularam o modelo dos três fatores. Esse modelo, segundo os autores, poderia explicar significativamente os retornos das ações. Os fatores de risco são o mercado, o tamanho da empresa e o índice book-to-market ou B/M. Costa Jr. e Neves (2000) e Málaga e Securato (2004) testaram e validaram esse modelo para o 
mercado brasileiro, encontrando evidências da importância dos fatores de risco adicionais ao beta.

De acordo comFama eFrench (1996; 2004), o modelo de três fatores captura a maior parte das anomalias não assimiladas pelo fator mercado, exceto a anomalia denominada efeito momento. Um grande número de estudos, iniciados por Jegadeesh e Titman (1993), demonstrou que estratégias de momento, que envolvem a compra (venda) de ações que tiveram um bom (mau) desempenho nos últimos 12 meses, tendem a produzir retornos anormais positivos durante o ano subsequente. Os resultados de Jegadeesh e Titman (1993) foram corroborados por Fama eFrench (1996) e Jegadeesh e Titman (2001) para o mercado norte-americano, por Rouwenhorst (1998) para o mercado europeu, por Rouwenhorst (1999) para os mercados emergentes. Para o mercado brasileiro, destacam-se os trabalhos de Lemos e Costa Jr. (1995) e Mussa et al. (2007).

Uma vez identificado o fator de risco momento, alguns estudos, sendo pioneiro o trabalho de Carhart (1997), passaram a adicioná-lo ao modelo dos três fatores de Fama eFrench (1993), construindo o que ficou conhecido como modelo dos quatro fatores. Em seu estudo, Carhart (1997) encontrou evidências empíricas para afirmar a superioridade do modelo dos quatro fatores em relação ao modelo dos três fatores, na explicação dos retornos. Mussa, Santos e Famá (2007) testaram o modelo dos quatro fatores no mercado acionário brasileiro, também encontrando evidências de sua validação e superioridade em relação ao CAPM e ao modelo dos três fatores.

Diante do sucesso empírico dos modelos dos três e dos quatro fatores, diversos autores, entre eles Jensen, Johnson e Mercer (1996; 1997), Chan, Karceski e Lakonishok (1998), e L'Her, Masmoudi e Suret (2004), passaram a buscar as causas da existência e da intensidade dos prêmios por esses fatores de risco adicionais ao beta.

Jensen, Johnson e Mercer (1996; 1997) evidenciaram que o ambiente de política monetária, expansiva ou restritiva, exerce importante influência no comportamento dos fatores de risco tamanho e índice $\mathrm{B} / \mathrm{M}$, no mercado acionário norte-americano.

Por sua vez, Chan, Karceski e Lakonishok (1998) investigaram a regularidade no comportamento dos fatores tamanho e índice $\mathrm{B} / \mathrm{M}$ por meio da análise da relação existente entre os prêmios por esses fatores de risco e os períodos de alta e baixa do mercado acionário. Os autores observaramque 
as condições do mercado influenciam o comportamento dos fatores nos mercados acionários norte-americano, japonês e inglês.

Com base nos dois estudos anteriores, L'Her, Masmoudi e Suret (2004) verificaram que as condições tanto do mercado acionário como da política monetária exercem influência significativa no comportamento dos fatores tamanho, índice $\mathrm{B} / \mathrm{M}$ e momento, no mercado acionário canadense.

Com base no que foi exposto, o objetivo do presente trabalho é investigar o comportamento dos fatores de risco tamanho, índice $\mathrm{B} / \mathrm{M} \mathrm{e}$ momento, no mercado acionário brasileiro, em condições de i) alta e baixa do mercado acionário e ii) expansão e retração monetária. A amostra é constituída por todas as ações listadas na Bolsa de Valores do Estado de São Paulo (Bovespa), no período de junho de 1995 a junho de 2007. A metodologia de testes empregada é essencialmente a mesma utilizada por Fama eFrench (1993) na construção das carteiras e fatores de risco.

A pesquisa destaca-se no Brasil por abordar um assunto importante para a comunidade acadêmica, à medida que agrega evidências do comportamento de alguns fatores de risco mais discutidos na literatura internacional. Além disso, por tratar de prêmios e retornos, o trabalho traz importantes contribuições para as estratégias de investimento, seja para pessoas físicas ou jurídicas.

O artigo está organizado da seguinte forma: a Seção 2 explica a metodologia utilizada no estudo, a Seção 3 aborda as análises dos resultados obtidos e, finalmente, a Seção 4 trata das considerações finais.

\section{Metodologia}

\subsection{Os Fatores de Risco}

Os fatores abordados nesta pesquisa são: o tamanho da empresa, definido pelo valor de mercado do patrimônio líquido; o índice book-to-market ou $\mathrm{B} / \mathrm{M}$, definido pela relação entre o valor contábil e de mercado do patrimônio líquido; e o momento, definido pelo desempenho acumulado dos retornos das ações. Esses fatores, adicionados ao beta de mercado do CAPM, compõem o modelo dos quatro fatores, batizado por Carhart (1997), que pode ser representado pela seguinte expressão matemática: 


$$
R_{c i, t}-R_{l r t}=a+b\left[R_{m t}-R_{l r t}\right]+s\left[S M B_{t}\right]+h\left[H M L_{t}\right]+w[\text { WinMLost }]+e_{i, t}
$$

Em que, $R_{C i, t}$ é oretorno da carteira $i$, no mês $t ; R_{m t}$ é oretorno da carteira de mercado no mês $t ; R_{l r t}$ é o retorno do ativo livre de risco no mês $t$; $S M B_{t} e ́$ o prêmio pelo fator tamanho no mês $t$ (SmallMinus Big ou a diferença entre a média de retorno das ações de empresas de pequeno porte e a média de retorno das ações de empresas de grande porte); $H M L_{t}$ é o prêmio pelo fator B/M no mês t (High MinusLow ou a diferença entre a média de retorno das ações com alto índice $\mathrm{B} / \mathrm{M}$ e a média de retorno das ações com baixo índice

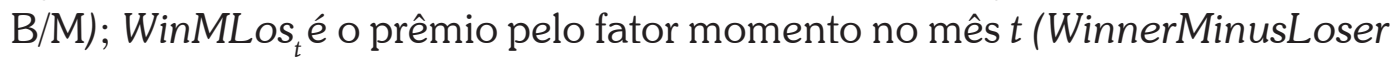
ou a diferença entre a média de retorno das ações de empresas que tiveram um alto desempenho passado e a média de retorno das ações de empresas que tiveram um baixo desempenho passado); $e_{i, t}=$ resíduo do modelo referente a carteira $i$ no mês $t$.

\subsection{Amostra}

A amostra analisada foi composta por todas as ações listadas na Bovespa entre $1^{\circ}$ de junho de 1995 e 30 de junho de 2007. Utilizou-se esse período de tempo devido à maior estabilidade da economia brasileira após o Plano Real. Assim, dados anteriores poderiam conter distorções.

Foram efetuadas as seguintes exclusões:

a) ações que não apresentavam cotações mensais consecutivas para o período de 12 meses anteriores ou 12 meses posteriores ao de formação das carteiras. Os meses anteriores foram necessários para o cálculo do fator momento e os posteriores para o cálculo do retorno das ações, que foram utilizados para a obtenção dos prêmios pelos fatores de risco;

b) ações sem valor de mercado em 31 de dezembro e 30 de junho, com tolerância de 23 dias;

c) ações de empresas que não possuíam Patrimônio Líquido positivo em dezembro;

d) empresas financeiras, devido a seu alto grau de endividamento, característico do setor. A exclusão decorre da influência que o endividamento tem sobre o índice $\mathrm{B} / \mathrm{M}$ e do fato do endividamento 
de empresas financeiras não ter o mesmo significado do endividamento de empresas não-financeiras;

e) ademais, para que não houvesse distorções do índice $\mathrm{B} / \mathrm{M}$ em empresas que possuíam ações de classe $\mathrm{ON}$ e PN, o valor de mercado para cálculo do índice foi obtido pelo somatório dos valores de mercado das ações $\mathrm{ON}$ e PN, mesmo que uma das duas classes de ações não tenha permanecido na amostra. Caso a ação não tenha apresentado valor de mercado em junho, com tolerância de 23 dias, para uma das duas classes de ações, ambas foram excluídas do estudo.

Diante do tratamento detalhado anteriormente, nota-se que a amostra final desta pesquisa não está livre do viés de sobrevivência. Porém, esse efeito está diminuído pelo fato das carteiras serem reformuladas a cada 12 meses e não mantidas iguais durante todo o período (de 1995 a 2007).

\subsection{Metodologia para Determinação dos Prêmios pelos Fatores de Risco}

\subsection{Preparação das Carteiras}

Em junho de cada ano t, de 1995 a 2007, todas as ações da amostra foram classificadas de acordo com o índice $\mathrm{B} / \mathrm{M}$ das empresas que a representam. $\mathrm{O}$ índice foi calculado conforme a equação (2). A amostra foi então separada em três grupos: $30 \%$ inferiores (Low), $40 \%$ médios (Medium) e 30\% superiores (High), de acordo com o valor do índice B/M. Utilizou-se o mês de junho, pois, conforme Fama eFrench (1992), os valores de mercados neste mês já refletem todas as informações contábeis anteriores.

$$
B / M_{i, t}=\frac{V C_{P L, d e z(t-1)}}{V M_{P L, d e z}(t-1)}
$$

Em que,

$B / M_{i, t}$ é o índice $\mathrm{B} / \mathrm{M}$, calculado com dados de dezembro de $t-1$, utilizado para a formação das carteiras no ano; $V C_{P L, d e z(t-1)}$ é o valor contábil do patrimônio líquido em 31 de dezembro do ano $t-1 ; V M_{P L, d e z(t-1)}$ é o valor de mercado do patrimônio líquido em 31 de dezembro do ano $t$-1. 
Para esse cálculo foram somados os valores de mercado do patrimônio líquido das ações $\mathrm{ON}$ e PN.

Também em junho de cada ano $t$, todas as ações foram ordenadas de acordo com o valor de mercado de junho das empresas representadas, conforme descrito na equação (3). O valor da mediana foi utilizado para dividir a amostra em dois grupos: B (Big) e S (Small), contendo as empresas de maior e menor valor de mercado, respectivamente.

$$
V M_{i, t}=\sum P_{y, i, t} \times N_{y, i, t}
$$

Em que,

$V M_{i, t}$ é o valor de mercado da empresa $i$ no momento $t ; P_{y, i, t}$ é o preço da ação do tipo $y$, da empresa $i$ no momento $t, N_{y, i, t}$ é o número de ações do tipo $y$, da empresa $i$, no momento $t$.

Neste mesmo mês, todas as ações foram ordenadas de acordo com o desempenho acumulado do retorno no período $t-2$ e $t$-12. Dessa forma, foi considerada a estratégia de momento de um ano de Jegadeesh e Titman (1993) e Carhart (1997), calculada de acordo com o desempenho dos últimos 12meses, desconsiderando o mês mais recente. Na sequência, a amostra foi separada pelo valor mediano em dois grupos: Los (Losers) e Win (Winners), contendo as empresas de piores e melhores retornos históricos acumulados, respectivamente.

Em junho de cada ano t,após as três ordenações anteriores, construíram-se 12 carteiras, decorrentes da interseção dos diversos grupos criados. As 12 carteiras estão descritas no Quadro 1 a seguir:

\begin{tabular}{|l|l|}
\hline \multicolumn{1}{|c|}{ Carteira } & \multicolumn{1}{|c|}{ Descrição } \\
\hline H/B/Los & $\begin{array}{l}\text { (High, Big andLoser) - ações com alto índice B/M, alto valor de mercado e } \\
\text { baixo desempenho passado. }\end{array}$ \\
\hline H/B/Win & $\begin{array}{l}\text { (High, Big andWinner) - ações com alto índice B/M, alto valor de mercado e } \\
\text { alto desempenho passado. }\end{array}$ \\
\hline H/S/Los & $\begin{array}{l}\text { (High, SmallandLoser) - ações com alto índice B/M, baixo valor de mercado e } \\
\text { baixo desempenho passado. }\end{array}$ \\
\hline H/S/Win & $\begin{array}{l}\text { (High, SmallandWinner) - ações com alto índice B/M, baixo valor de mercado } \\
\text { e alto desempenho passado. }\end{array}$ \\
\hline L/B/Los & $\begin{array}{l}\text { (Low, Big andLoser) - ações com baixo índice B/M, alto valor de mercado e } \\
\text { baixo desempenho passado. }\end{array}$ \\
\hline
\end{tabular}




\begin{tabular}{|c|c|}
\hline Carteira & Descrição \\
\hline $\mathrm{L} / \mathrm{B} / \mathrm{Win}$ & $\begin{array}{l}\text { (Low, Big andWinner) - ações com baixo índice B/M, alto valor de mercado e } \\
\text { alto desempenho passado. }\end{array}$ \\
\hline L/S/Los & $\begin{array}{l}\text { (Low, SmallandLoser) - ações com baixo índice B/M, baixo valor de mercado } \\
\text { e baixo desempenho passado. }\end{array}$ \\
\hline L/S/Win & $\begin{array}{l}\text { (Low, SmallandWinner) - ações com baixo índice } \mathrm{B} / \mathrm{M} \text {, baixo valor de merca- } \\
\text { do e alto desempenho passado. }\end{array}$ \\
\hline $\mathrm{M} / \mathrm{B} / \mathrm{Los}$ & $\begin{array}{l}\text { (Medium, Big andLoser) - ações com médio índice } \mathrm{B} / \mathrm{M} \text {, alto valor de mercado } \\
\text { e baixo desempenho passado. }\end{array}$ \\
\hline $\mathrm{M} / \mathrm{B} / \mathrm{Win}$ & $\begin{array}{l}\text { (Medium, Big andWinner) - ações com médio índice } \mathrm{B} / \mathrm{M} \text {, alto valor de } \\
\text { mercado e alto desempenho passado. }\end{array}$ \\
\hline M/S/Los & $\begin{array}{l}\text { (Medium, SmallandLoser) - ações com médio índice B/M, baixo valor de } \\
\text { mercado e baixo desempenho passado. }\end{array}$ \\
\hline $\mathrm{M} / \mathrm{S} / \mathrm{Win}$ & $\begin{array}{l}\text { (Medium, SmallandWinner) - ações com médio índice B/M, baixo valor de } \\
\text { mercado e alto desempenho passado. }\end{array}$ \\
\hline
\end{tabular}

Quadro 1: Descrição das carteiras

Fonte: Elaborado pelos autores

De julho do ano $t$ a junho de $t+1$, calculou-se o retorno mensal de cada ação conforme demonstrado na equação (4).

$$
R_{i, t}=\frac{\operatorname{Div}_{i, t}}{P_{i, t-1}}+\frac{P_{i, t}-P_{i, t-1}}{P_{i, t-1}}
$$

Em que $R_{i, t}$ é o retorno da ação i para o mês $t$; Div $v_{i, t}$ é o dividendos da ação $i$ distribuídos durante o mês $t ; P_{i, t-1}$ é o valor da ação $i$ ao fim do mês t$1 ; P_{i, t}$ é o valor da ação $i$ ao final do mês $t$.

\subsubsection{Obtenção dos Prêmios pelos Fatores de Risco}

Os prêmios pelo fator de risco mercado foram obtidos utilizando a equação (5):

$$
\text { Prêmio de Mercado } \mathrm{t}=\left[\sum_{\mathrm{i}=1}^{\mathrm{n}} \frac{V M_{i, t}}{V M_{M t,}}\left(R_{i, t}\right)\right]-R f_{t}
$$

Em que, $R_{i, t}$ é o retorno da ação $i$, pertencente à carteira de mercado, no mês $t ; V M_{i, t}$ é o valor de mercado da ação $i$, ao final do mês $t ; V M_{M, t}$ é ovalor de 
mercado da carteira de mercado, considerando todas as ações da amostra, no mês $t ; R f_{t}$ é oretorno do ativo livre de risco, no mês $t$. Adotou-se o retorno mensal da caderneta de poupança como uma aproximação para a taxa livre de risco, conforme sugerido por Barros, Famá e Silveira (2003).

A equação (6) foi utilizada para cálculo dos prêmios pelo fator tamanho (SMB):

$$
S M B_{t}=\overline{R_{S, t}}-\overline{R_{B, t}}
$$

Em que, $S M B_{t}$ é o prêmio pelo fator tamanho, no mês $t ; R_{S, t}$ é oretorno médio mensal das seis carteiras $\mathrm{S} ; R_{\mathrm{B}, t}$ é oretorno médio mensal das seis carteiras B.

Os prêmios pelo fator índice $\mathrm{B} / \mathrm{M}$ (HML) foram calculados conforme a equação (7):

$$
H M L_{t}=\overline{R_{H, t}}-\overline{R_{L, t}}
$$

Em que, $H M L_{t}$ é oprêmio mensal pelo fator de risco $\mathrm{B} / \mathrm{M} ; R_{H, t}$ é oretorno médio mensal das quatro carteiras $\mathrm{H} ; R L_{, t}$ é oretorno médio mensal das quatro carteiras L.

Por sua vez, os prêmios pelo fator momento (WinMLos) foram obtidos utilizando a equação (8):

$$
\text { WinMLoSt }=\overline{R_{W I N, t}}-\overline{R_{L O S, t}}
$$

Em que, WinMLos é oprêmio mensal pelo fator de risco momento; $R_{\text {WIN,t }}$ é oretorno médio mensal das seis carteiras Win; $R_{L O S, t}$ é oretorno médio mensal das seis carteiras Los.

Todas as etapas descritas anteriormenteforam repetidas em junho de cada ano $t$, de 1995 a 2006.

\subsection{Metodologia para Separação da Amostra em Períodos de Alta e Baixa do Mercado Acionário e de Expansão e Restrição Monetária}

Seguindo Chan, Karceski e Lakonishok (1998) e L'Her, Masmoudi e Suret (2004), a separação da amostra em períodos de alta e baixa do mercado acionário foi feita da seguinte forma: 
a. Alta: meses em que o retorno da carteira de mercado foi superior ao retorno do ativo livre de risco (Caderneta de Poupança).

b. Baixa: meses em que o retorno da carteira de mercado foi inferior ao retorno do ativo livre de risco (Caderneta de Poupança).

Sabendo que a determinação da taxa de juros básica da economia é uma das principais ferramentas que um governo tem para conduzir sua política monetária, a variável utilizada no presente artigo para a segregação da amostra em períodos de política monetária expansiva e restritiva foi a taxa média mensal efetiva das LFTs (Letra Financeira do Tesouro). Essa foi também a estratégia adotada por LHer, Masmoudi e Suret (2004). Assim:

c. Expansão monetária: meses em que a taxa efetiva das LFTs foi inferior à média das taxas efetivas das LFTs dos 12 meses anteriores.

d. Restrição monetária: meses em que a taxa efetiva das LFTs foi superior à média das taxas efetivas das LFTs dos 12 meses anteriores.

A comparação da taxa do mês com a média das taxas dos últimos 12 meses foi motivada, segundo L'Her, Masmoudi e Suret (2004), pela necessidade de se evitar distorções dos dados por decisões dos bancos centrais que visam apenas o curto prazo.

\subsection{Coleta e Processamento de Dados}

Esta pesquisa utilizou-se de dados secundários extraídos do banco de dados da empresa de consultoria Economática. Os cálculos estatísticos foram processados utilizando o sistema estatístico Statistical Package for Social Sciences (SPSS), versão 13.

\section{Análise dos Dados e Resultados}

\subsection{Estatística Descritiva dos Prêmios pelos Fatores de Risco}

A Tabela 1 apresenta o prêmio mensal dos fatores de risco, o desviopadrão e o teste de $t$ de comparação de médias, considerando a amostra completa: 
Tabela 1:Prêmios mensais, desvio-padrão e teste $t$ dos prêmios pelos fatores de risco, sem qualquer segregação da amostra. Período: de julho de 1995 a junho de 2007

\begin{tabular}{lcccc}
\hline $\mathbf{N}=144$ observações & & & & \\
\hline Painel A & Rm-Rf & SMB & HML & WinMLos \\
\hline Média & $1,68 \%$ & $0,04 \%$ & $1,21 \%$ & $-0,05 \%$ \\
Desvio-padrão & $8,55 \%$ & $5,92 \%$ & $5,76 \%$ & $4,49 \%$ \\
$t$ & 2,36 & 0,07 & 2,52 & $-0,14$ \\
$p$-value de $t$ & $2,00 \%$ & $94,30 \%$ & $1,30 \%$ & $88,50 \%$ \\
\hline
\end{tabular}

Fonte: Elaborada pelos autores

O prêmio médio mensal de mercado foi de 1,68\%. Esse valor pode ser considerado estatisticamente diferente de zero, de acordo com o teste $t$ de Student, uma vez que o p-value é inferior a 5\%. Málaga e Securato (2004) encontraram 1,09\% como prêmio pelo fator mercado. Tal incremento de prêmio médio pode ter sido motivado pela diferença de período analisado entre os estudos, pois, de 2003 a 2007, a bolsa de valores brasileira teve um desempenho superior à média verificada de 1995 a 2002. Fama e French (1993) encontraram um prêmio de $0,43 \%$ para o mercado norte-americano.

Em relação ao fator tamanho, verificou-se um prêmio médio mensal praticamente nulo, de apenas $0,04 \%$. O baixo valor de te o $p$-value superior a $5 \%$ indicam que esse retorno não pode ser considerado estatisticamente diferente de zero. Assim, empresas pequenas não parecem oferecer retornos superiores aos retornos proporcionados por empresas de grande porte. Esse resultado corrobora os resultados de Málaga e Securato (2004), que chegaram a um retorno médio mensal negativo (-0,32\%), também estatisticamente não diferente de zero. Fama e French (1993) constataram um prêmio SMB de $0,27 \%$ ao mês para o mercado norte-americano, mas que também não pôde ser considerado estatisticamente diferente de zero.

Já, para o fator HML, este estudo encontrou um prêmio positivo de $1,21 \%$, estatisticamente diferente de zero. Esse percentual foi superior ao constatado por Málaga e Securato (2004), de 0,59\% e estatisticamente igual a zero. Fama e French (1993) encontraram um prêmio de 0,40\%, para o mercado norte-americano, também estatisticamente diferente de zero. 
Quanto ao fator WinMLos, não encontrou-se um prêmio, uma vez que a diferença entre o retorno das carteiras Win e Los foi negativa $(-0,05 \%)$. Assim, parece haver no Brasil uma relação inversa à observada nos estudos de Jegadeesh e Titman $(1993 ; 2001)$ para o mercado norte-americano e Rouwenhorst (1998) para o mercado europeu. A análise da estatística t, com $p$-valuesuperior a $5 \%$, indica que esse retorno não pode ser considerado estatisticamente diferente de zero. Jegadeesh e Titman (1993; 2001) chegaram a retornos estatisticamente diferente de zero para o mercado norte-americano utilizando a estratégia de 12 meses.

Rouwenhorst (1999), apesar de ter encontrado um prêmio positivo para o fator momento para a média dos países emergentes, chegou a um valor praticamente nulo $(0,01 \%)$ para o Brasil, que não pôde ser considerado estatisticamente diferente de zero. Já Liew e Vassalou (2000) chegaram a valores negativos para o prêmio pelo fator momento nos mercados italiano e japonês.

\subsection{O Comportamento dos Retornos nos Ambientes de Alta e de Baixa do Mercado Acionário}

A Tabela 2 traz o resumo estatístico dos prêmios mensais pelos fatores de risco considerando a separação da amostra em períodos de alta e de baixa do mercado acionário brasileiro: 
Tabela 2:Sumário estatístico dos retornos mensais dos quatro fatores, considerando a estratificação da amostra em períodos de alta e baixa do mercado acionário brasileiro. Período: de julho de 1995 a junho de 2007

\section{Painel A: períodos de baixa do mercado acionário}

\begin{tabular}{lcccc}
\hline $\mathbf{N}=\mathbf{5 3}$ observações & Rm-Rf & SMB & HML & WinMLos \\
\hline Média & $-6,39 \%$ & $3,20 \%$ & $0,63 \%$ & $0,50 \%$ \\
Desvio-padrão & $7,15 \%$ & $5,36 \%$ & $4,58 \%$ & $4,22 \%$ \\
$t$ & $-6,50$ & 4,34 & 0,99 & 0,86 \\
p-value de $t$ & $0,00 \%$ & $0,00 \%$ & $32,50 \%$ & $39,20 \%$
\end{tabular}

Painel B: períodos de alta do mercado acionário

\begin{tabular}{lcccc}
\hline $\mathbf{N}=91$ observações & Rm-Rf & SMB & HML & WinMLos \\
\hline Média & $6,38 \%$ & $-1,81 \%$ & $1,55 \%$ & $-0,38 \%$ \\
Desvio-padrão & $5,09 \%$ & $5,45 \%$ & $6,35 \%$ & $4,63 \%$ \\
$t$ & 11,97 & $-3,16$ & 2,33 & $-0,78$ \\
p-value de $t$ & $0,00 \%$ & $0,20 \%$ & $2,20 \%$ & $43,90 \%$ \\
\hline
\end{tabular}

Fonte: Elaborada pelos autores

O excesso de retorno da carteira de mercado em relação ao ativo livre de risco apresentou-se positivo em grande parte do período, ou seja, em $63 \%$ das observações. Com exceção do fator índice B/M (HML), os retornos médios mostraram-se notavelmente maiores nos períodos de baixa. Chan, Karseski e Lakonishok (1998) sugerem que esse fato esteja relacionado às estratégias defensivas dos investidores.

Com a separação da amostra, o prêmio pelo fator tamanho (SMB) passou a ser estatisticamente diferente de zero ( $p$-values do teste inferiores a $5 \%$ ) nos dois grupos: períodos de alta e baixa do mercado acionário, trazendo evidências de que as condições do mercado exercem influência sobre o comportamento desse fator. Porém, o prêmio apenasfoi positivo nos períodos de baixa (3,20\%). Chan, Karseski e Lakonishok (1998) chegaram a conclusões semelhantes.

O prêmio pelo fator índice $\mathrm{B} / \mathrm{M}$ (HML) se mostrou estatisticamente diferente de zero somente para as situações de alta do mercado acionário. O prêmio encontrado foi de $1,55 \%$ nos períodos de alta e $0,63 \%$ nos períodos de baixa. Chan e Chen (1991) e Fama eFrench (1992; 1993; 1995; 2004) 
sugerem que a inclusão do fator HML nos modelos de precificação seria suportada pelo fato das ações com alto índice $\mathrm{B} / \mathrm{M}$ apresentarem, de forma persistente, baixos retornos sobre seus ativos, o que permitiria o surgimento de um risco adicional - conhecido como distressfactor, que justificaria a posterior observação de maiores retornos. Dessa forma, os resultados na presente pesquisa são consistentes com os argumentos de Chan e Chen (1991) e de Fama e French $(1992 ; 1993 ; 1995 ; 2004)$, uma vez que se observou uma relação positiva entre altos índices $\mathrm{B} / \mathrm{M}$ e os retornos subsequentes. L'Her, Masmoudi e Suret (2004) encontraram evidências contrárias no mercado canadense, uma vez que observaram altos retornos por esse fator em períodos de baixa do mercado acionário.

Já o prêmio pelo fator de risco momento, WinMLos, se mostrou positivo apenas para os períodos de baixa do mercado acionário. Em nenhuma das situações do mercado, o prêmio por esse fator se mostrou estatisticamente diferente de zero. Esses resultados vão de encontro aos verificados por LHer, Masmoudi e Suret (2004) no mercado canadense. Os autores chegaram a prêmios positivos e estatisticamente diferentes de zero para o fator momento em ambos os contextos (1,05\% para os períodos de baixa e $1,57 \%$ para os períodos de alta). Do ponto de vista econômico, observou-se um retorno positivo $(0,50 \%)$ no ambiente de baixa.

\subsection{O Comportamento dos Retornos nas Situações de Expansão e Restrição Monetária}

Jensen, Johnson e Mercer (1996; 1997) sugeriram que a percepção de risco dos investidores se modifica com alterações na política monetária. Os autores demonstraram que o prêmio por ações de pequenas empresas e com baixos índices $\mathrm{B} / \mathrm{M}$ são economicamente e estatisticamente significantes somente em períodos de expansão da política monetária, no mercado norteamericano.

LHer, Masmoudi e Suret (2004), aplicando metodologia semelhante para o mercado acionário canadense, encontraram que os prêmios SMB e HML não são significativos em ambiente de restrição monetária, comoeles são altamente significantes em ambiente de política monetária expansiva. A estratificação teve pequeno impacto no comportamento do fator momento, que se mostrou significativo em ambos os contextos. 
A Tabela 3 traz o sumário estatístico dos retornos mensais dos quatro fatores de risco, considerando a separação da amostra em períodos de política monetária expansiva e restritiva, para o mercado acionário brasileiro.

Tabela 3:Sumário estatístico dos retornos mensais dos quatro fatores, considerando a estratificação da amostra em períodos de expansão e restrição monetária. Período: de julho de 1995 a junho de 2007

\section{Painel A: períodos de política monetária restritiva}

\begin{tabular}{lcccc}
\hline $\mathbf{N}=\mathbf{4 7}$ observações & Rm-Rf & SMB & HML & WinMLos \\
\hline Média & $2,24 \%$ & $-1,15 \%$ & $2,08 \%$ & $0,04 \%$ \\
Desvio-padrão & $8,36 \%$ & $6,67 \%$ & $6,66 \%$ & $5,46 \%$ \\
$t$ & 1,83 & $-1,18$ & 2,14 & 0,05 \\
p-value de $t$ & $7,30 \%$ & $24,50 \%$ & $3,70 \%$ & $96,30 \%$ \\
\hline Painel B: períodos de política monetária expansiva & & & \\
\hline $\mathbf{N}=\mathbf{9 7}$ observações & Rm-Rf & SMB & HML & WinMLos \\
\hline Média & $1,41 \%$ & $0,61 \%$ & $0,79 \%$ & $-0,10 \%$ \\
Desvio-padrão & $8,67 \%$ & $5,46 \%$ & $5,25 \%$ & $3,96 \%$ \\
$t$ & 1,61 & 1,10 & 1,48 & $-0,24$ \\
p-value de $t$ & $11,20 \%$ & $27,60 \%$ & $14,30 \%$ & $80,70 \%$ \\
\hline
\end{tabular}

Fonte: Elaborada pelos autores

Nota-se que o único prêmio que se mostrou estatisticamente diferente de zero foi o HML na situação de expansão monetária, com $p$-value inferior a $5 \%$. Esse resultado foi diferente do verificado no mercado norte-americano por Jensen, Johnson e Mercer (1996; 1997), que encontraram prêmios estatisticamente significativos para os fatores SMB e HML, nos períodos de expansão monetária. L'Her, Masmoudi e Suret (2004) chegaram a prêmios estatisticamente significativos para todos os fatores nos períodos de expansão monetária e apenas para o fator momento no contexto de retração monetária.

Do ponto de vista econômico, verifica-se que o prêmio pelo fator SMB parece ter sofrido influência da política monetária, semelhante ao que foi verificado por Jensen, Johnson e Mercer (1996; 1997) e por LHer, Masmoudi e Suret (2004), uma vez que o retorno foi maior e positivo no contexto de 
expansão monetária $(0,61 \%)$. Ressalta-se que, sem a separação amostral, o prêmio SMB havia se apresentado praticamente nulo (0,04\% ao mês).

O prêmio pelo fator HML também se mostrou positivo nas duas situações de política monetária. Porém, no Brasil, o prêmio foi maior na fase de restrição monetária. Já o prêmio pelo fator momento (WinMLos), da mesma forma que ocorreu na pesquisa de L'Her, Masmoudi e Suret (2004), não parece sofrer influência significativa das condições de política monetária, uma vez que nos dois grupos, de expansão e retração monetária, o prêmio permaneceu muito próximo de zero $(0,04 \%$ no contexto de restrição monetária e $-0,10 \%$ no contexto de expansão monetária).

\section{Considerações Finais}

O objetivo do presente artigo foi investigar a regularidade no comportamento dos fatores de risco tamanho, índice $\mathrm{B} / \mathrm{M}$ e momento, no mercado acionário brasileiro, em ambientes de i) alta e baixa do mercado acionário $e$ ii) expansão e retração monetária. A amostra foi constituída por todas as ações listadas na Bolsa de Valores do Estado de São Paulo - BOVESPA, no período de junho de 1995 a junho de 2007. A metodologia de testes empregada foi essencialmente a mesma utilizada por Fama eFrench (1993) na construção das carteiras e fatores de risco.

A análise inicial, sem considerar quaisquer segregações da amostra, encontrou prêmios médios mensais de $1,68 \%, 0,04 \%, 1,21 \%$ e - $0,05 \%$, respectivamente pelos fatores de risco mercado, tamanho, índice $\mathrm{B} / \mathrm{M}$ e momento. Os prêmios praticamente nulos pelos fatores tamanho e índice $\mathrm{B} / \mathrm{M}$ contrastam os resultados encontrados por Fama e French (1993) e Jegadeesh e Titman (1993; 2001) no mercado norte-americano.

Na mesma linha dos resultados encontrados por Chan, Karceski e Lakonishok (1998), observou-se a influência das condições de mercado no comportamento do fator de risco tamanho (SMB). O prêmio SMB se mostrou positivo $(3,20 \%)$ durante os períodos de baixa do mercado acionário e negativo $(-1,81 \%)$ durante os períodos de alta. Por outro lado, contrariando Chan, Karceski e Lakonishok (1998) e L'Her, Masmoudi e Suret (2004), o prêmio HML se mostrou mais forte no contexto de alta do mercado acionário, corroborando a suposição de Chan e Chen (1991) e de Fama e French (1992; 1993; 1995; 2004) de que esse prêmio está associado ao risco adicional 
conhecido por distressfactor. Já o prêmio pelo fator momento (WinMLos) parece ser menos afetado pelas condições do mercado.

Com a segregação da amostra em períodos de expansão e restrição monetária, apenas o fator HML, no ambiente de política monetária restritiva, se mostrou estatisticamente diferente de zero. Somente o prêmio pelo fator SMB apresentou-se maior nos períodos de expansão monetária do que nos períodos de restrição, contrariando as evidências de Jensen, Johnson e Mercer (1996; 1997) e L'Her, Masmoudi e Suret (2004). Do ponto de vista econômico, a influência sofrida pelo fator tamanho (SMB) ocorreu de forma semelhante à verificada por Jensen, Johnson e Mercer (1996; 1997) e L'Her, Masmoudi e Suret (2004).

Este artigo contribui para a teoria de finanças, à medida que agrega evidências do comportamento, no mercado acionário brasileiro, de alguns dos principais fatores de risco estudados pela comunidade acadêmica. Além disso, traz contribuições para a polêmica discussão a respeito das causas da existência e da intensidade desses fatores. Do ponto de vista prático, os resultados podem auxiliar os investidores, sejam pessoas físicas ou jurídicas, na definição das estratégias de gerenciamento de suas carteiras de investimento.

\section{The Influence of Conditions of the Stock Market and Monetary Policy on the Behaviorof Risk Indicators Size, Book-to-market Ratio and Momentum, on the Brazilian Stock Market}

\section{Abstract}

Last years, empirical tests of APT (Arbitrage Pricing Theory) models have been intensified on the national and international literature, mainly using firm's characteristics to construct risk factors in addition to the market beta. The FamaFrench 3-factors model and the Carhart 4-factors model are two samples intensively tested of this type of models, with evidences of relative success. On this scenario, it is important to deepen the studies of the factor's behavior that compose these models. Following the way of international researches, the purpose of this article is to investigate the behavior of the factors: size, book-to-market ratio and momentum, on the Brazilian stock market, in conditions of i) up and down markets ii) expansive and restrictive monetary policy. The sample was composed by all stocks listed on 
BOVESPA, from June of 1995 to June of 2007. The methodology was the same used by Fama \& French (1993) to construct the portfolios and risk factors. The results indicated that the stock market and monetary environment influence the regularities in the factor's behavior, on the Brazilian Stock markets.

Key words: CAPM. APT. Risk Factors.

\section{Referências}

BANZ, R. The relationship between return and market value of common stock. Journal of Financial Economics, Amsterdam: North Holland, v. 9, n. 1, p. 3-18, 1981.

BARROS, L. de C.; FAMÁ, R.; SILVEIRA, H. P. Aspectos da teoria de portfólio em mercados emergentes: uma análise de aproximações para a taxa livre de risco no Brasil. SEMEAD, 6., São Paulo. Anais... Universidade de São Paulo, 2003.

BASU, S. Investment performance of common stocks in relation to their price earnings ratios: a test of the efficient market hypothesis. Journal of Finance, Chicago: American Finance Association, v. 32, n. 3, p. 663-682, 1977.

BLACK, F.; JENSEN, M.; SCHOLES, M. The capital asset pricing model: some empirical testes. In: JENSEN, M. C. (Org.). Studies in the Theory of

Capital Markets, New York: Praeger, 1972.

CARHART, M. M. On persistence in mutual fund performance. Journal of Finance, Chicago: American Finance Association, v. 52, n. 1, p.57-82, 1997.

CHAN, K. C.; CHEN, N. F. Structural and return characteristics of small and large firms. The Journal of Finance, Chicago: American Finance Association, v. 46, n. 4, p. 1467-1484, 1991.

CHAN, L. K. C.; KARCESKI, J.; LAKONISHOK, J. The risk and return from factors. The Journal of Financial and Quantitative Analysis, Seattle: University of Washington, v. 32, n. 2, p. 159-188, 1998.

COSTA JR, N.C.A.; NEVES, M.B.E. Variáveis fundamentalistas e os retornos das ações. Revista Brasileira de Economia, Rio de Janeiro: Fundação Getúlio Vargas, v. 54, n. 1, 2000. 
FAMA, E. F.; FRENCH, K. The cross-section of expected stock returns. Journal of Finance, Chicago: American Finance Association, v. 47, n. 2, p. 427-465, 1992.

. Common risk factors in the returns on stocks and bonds. Journal of Financial Economics, Amsterdam: North Holland, v. 33, n. 1, p.3-56, 1993.

. Size and book-to-market factors in earnings and returns. Journal of Finance, Chicago: American Finance Association, v. 50, n. 1, p. 131-155, March 1995.

. Multifactor explanation of asset pricing anomalies. Journal of Finance, Chicago: American Finance Association, v. 56, n. 1, p.55-84, 1996.

. The capital asset pricing model: theory and evidence. Journal of Economic Perspectives, Nashville: American Finance Association, v. 18, n. 3, p. 25-46, 2004.

FAMA, E. F; MACBETH, J. D. Risk, return and equilibrium: empirical testes. Journal of Political Economy, Chicago: The University of Chicago Press, v. 81, n. 3 , p. $607-636,1973$.

JEGADEESH, N.; TITMAN, S. Returns to buying winners and selling losers: implications for stock market efficiency. Journal of Finance, Chicago: American Finance Association, v. 48, n. 1, p. 65-91, 1993.

. Profitability of momentum strategies: an evaluation of alternative explanations. Journal of Finance, Chicago: American Finance Association, v. 56, n. 2, p. 699-720, 2001.

JENSEN, G. R.; JOHNSON, R. R.; MERCER, J. M. Business conditions, monetary policy, and expected security returns. Journal of Financial Economics, Amsterdam: North Holland, v. 40, n. 2, p. 213-237, 1996.

. New evidence on size and price-to-book effects in stock returns.

Financial Analysts Journal, Charlottesville: CFA Institute Publication v. 53, n. 6, p. 34-42, 1997.

LHER, J. F.; MASMOUD, T.; SURET, J. M. Evidence to support the four-factor pricing model from the Canadian stock market. Journal of International Financial Markets, Institutions and Money, Amsterdam: Elsevier Science Publishers, v. 14, n. 4, p. 313-328, 2004. 
LAKONISHOK, J.; SHAPIRO, A. C. Systematic risk, total risk and size as determinants of stock market returns. Journal of Banking and Finance, Amsterdam: Elsevier Science Publishers, v. 10, n. 1, p. 115-132, 1986.

LEMOS, M. O. ; COSTA JR., N. C. A. A Sobre-Reação a curto prazo no mercado de capitais brasileiro. RAC. Revista de Administração

Contemporânea, Curitiba: Anpad, v. 1, n. 2, p. 291-309, 1995.

LIEW, J.; VASSALOU, M. Can book-to-market, size and momentum be risk factors that predict economic growth? Journal of Financial Economics, Amsterdam: North Holland, v. 57, n. 2, p. 221-245, 2000.

MÁLAGA, F. K.; SECURATO, J. R. Aplicação do modelo dos três fatores de Fama \& French no mercado acionário brasileiro - um estudo empírico do período 1995-2003. In: ENCONTRO ANUAL DA ASSOCIAÇÃO NACIONAL DOS PROGRAMAS DE PÓS-GRADUAÇÃO EM ADMINISTRAÇÃO, 28., 2004, Curitiba. Anais... Curitiba: ANPAD, 2004.

MARKOWITZ, H. M. Portfolio Selection. Journal of Finance, Chicago: American Finance Association, v. 7, p. 77-91, 1952.

MUSSA, A. et. al. A estratégia de momento de Jegadeesh e Titman e suas implicações para a hipótese de eficiência do mercado acionário brasileiro. In: SEMINÁRIOS EM ADMINISTRAÇÃO FEA/USP - SEMEAD, 10., 2007, São Paulo. Anais... São Paulo: USP, 2007.

MUSSA, A. SANTOS, J.O., FAMA, R. A adição do fator de risco momento ao modelo de precificação de ativos dos três fatores de Fama \& French, aplicado ao mercado acionário brasileiro. In: CONGRESSO USP DE CONTROLADORIA E CONTABILIDADE, 7., 2007, São Paulo. Anais... São Paulo: USP, 2007.

ROUWENHORST, K. G. International momentum estrategies. Journal of Finance, Chicago: American Finance Association, v. 53, n. 1, p. 267-284, 1998.

ROUWENHORST, K. G. Local return factors and turnover in emerging stock markets. Journal of Finance, Chicago: American Finance Association, v. 54, n. 4, p. 1439-1464, 1999.

SHARPE, W. F. Capital asset prices: a theory of market equilibrium under conditions of risk. Journal of Finance, Chicago: American Finance Association, v. 19, p. 425-443, 1964. 
STATTMAN, D. Book values and stock returns. The Chicago MBA:

A Journal of Selected Papers, Chicago, v. 4, p. 25-45, 1980.

TOBIN, J. Liquidity preference as behavior toward risk. The Review of

Economic Studies, Stockholm: Oxford University Press v. 25, n. 2, p. 65-86, 1958. 